\title{
Effect of evaporation-induced osmotic changes in culture media in a dry-type incubator on clinical outcomes in in vitro fertilization-embryo transfer cycles
}

\author{
Hee-Jun Chi ${ }^{1}$, Jun-Sang Park', Chang-Seok Yoo ${ }^{1}$, Su-Jin Kwak' ${ }^{1}$, Ho-Jeong Son ${ }^{1}$, Seok-Gi Kim', Chae-Hee Sim ${ }^{1}$, \\ Kyeong-Ho Lee ${ }^{1}$, Deog-Bon Koo ${ }^{2}$ \\ 'Mamapapa and Baby Ob/Gy Clinic, Ulsan; ${ }^{2}$ Institute of Infertility, Daegu University, Daegu, Korea
}

Objective: This study investigated whether adding outer-well medium to inhibit osmotic changes in culture media in a dry-type incubator improved the clinical outcomes of in vitro fertilization-embryo transfer (IVF-ET) cycles.

Methods: In culture dishes, the osmotic changes in media $(20 \mu \mathrm{L}$ )-covered oil with or without outer-well medium (humid or dry culture conditions, respectively) were compared after 3 days of incubation in a dry-type incubator. One-step (Origio) and G1/G2 (Vitrolife) media were used.

Results: The osmotic changes in the dry culture condition (308 mOsm) were higher than in the humid culture conditions (285-290 mOsm) after 3 days of incubation. In day 3 IVF-ET cycles, although the pregnancy rate did not significantly differ between the dry (46.2\%) and humid culture (51.0\%) groups, the rates of abortion and ongoing pregnancy were significantly better in the humid culture group (1.5\% and $49.5 \%$, respectively) than in the dry culture group ( $8.3 \%$ and $37.8 \%$, respectively, $p<0.05)$. In day 5 IVF-ET cycles, the abortion rate was significantly lower in the humid culture group (2.2\%) than in the dry culture group $(25.0 \%, p<0.01)$, but no statistically significant difference was observed in the rates of clinical and ongoing pregnancy between the dry (50.0\% and $25.0 \%$, respectively) and humid culture groups (59.5\% and $57.3 \%$, respectively) because of the small number of cycles.

Conclusion: Hyperosmotic changes in media occurred in a dry-type incubator by evaporation, although the medium was covered with oil. These osmotic changes were efficiently inhibited by supplementation of outer-well medium, which resulted in improved pregnancy outcomes.

Keywords: Clinical outcomes; Dry-type incubator; Evaporation; Osmotic change; Outer-well medium

\section{Introduction}

Even brief exposure of preimplantation mouse embryos to high-os-

Received: January 31, 2020 · Revised: June 08, 2020 · Accepted: June 15, 2020 Corresponding author: Hee-Jun Chi

Mamapapa and Baby Ob/Gy Clinic, 247 Samsan-ro, Nam-gu, Ulsan 44707, Korea Tel: +82-52-258-6006 Fax: +82-52-258-6026 E-mail: hanna129@hanmail.net

This is an Open Access article distributed under the terms of the Creative Commons Attribution Non-Commercial License (http://creativecommons.org/licenses/by-nc/4.0/) which permits unrestricted non-commercial use, distribution, and reproduction in any medium, provided the original work is properly cited. molality culture medium ( $>300 \mathrm{mOsm} / \mathrm{kg}$ ) in the absence of osmolytes resulted in impaired development [1-3]. The detrimental effect of hyperosmolality has also been reported in the development of various mammalian embryos, including murine [4-6], rat [7], porcine $[8,9]$, and bovine $[10,11]$ embryos. Moreover, in vitro two-cell blocks of mouse embryos were significantly alleviated by culturing them in low-osmolarity ( $250 \mathrm{mOsm}$ ) medium compared to high-osmolality ( > 300 mOsm) medium [12]. Biggers et al. [3] reported that when the $\mathrm{NaCl}$ concentration was increased in the medium, the intracellular $\mathrm{Na}^{+} / \mathrm{K}^{+}$ratio dramatically increased, which was detrimen- 
tal to mouse embryo development. The detrimental effects of hyperosmolality occur by triggering cell shrinkage, oxidative stress, protein carbonylation, mitochondrial depolarization, DNA damage, cell cycle arrest, and apoptosis [13,14].

From a different point of view, an increase in extracellular osmolality can promote water flux out of the cell, triggering cell shrinkage and intracellular dehydration [15]. Intracellular water loss interferes with many cellular functions, including DNA synthesis and repair, transcription, protein translation and degradation, and mitochondrial function. As a result, cell cycle progression and cell proliferation are arrested [13]. Dry-type incubators are now widely used instead of humid-type incubators due to the development of infrared $\mathrm{CO}_{2}$ sensors [16] and the low possibility of microorganism overgrowth [17]. In addition, dry-type incubators have smaller culture chambers than humid-type incubators, is advantageous in terms of the short recovery time for gas and temperature after the door is opened. However, concerns about the possible change in the osmolality of the medium by evaporation remain a reason why clinicians may hesitate to use a dry-type incubator. Although overlying culture dishes with oil could inhibit the shift in the osmolality of the medium, whether it completely eliminates the change in osmolality remains controversial [18]. Recently, human embryos cultured in dry-type incubators showed significantly lower implantation and clinical pregnancy rates than those cultured in humid-type incubators [18].

The present study was performed to compare osmotic changes in culture media covered in oil in various types of culture dishes, and to investigate whether a beneficial effect on clinical outcomes in in vitro fertilization-embryo transfer (IVF-ET) cycles could be obtained by supplementation with outer-well medium to inhibit osmotic changes in culture media in a dry-type incubator.

\section{Methods}

This retrospective study was approved by the Institutional Review Board of Mamapapa and Baby Clinic (IRB No. 2019-10-01), and was conducted from August 2018 to August 2019.

\section{Patients}

In total, 796 IVF-ET cycles in 673 patients were analyzed in the present study. Twelve patients who underwent their first 3-day IVFET cycles using a cell culture dish, but failed to show implantation or ongoing pregnancy, completed their second IVF-ET cycles using a GPS dish to compare the clinical outcomes between cell culture and GPS dish cycles. After a comparison of these 12 patients, we changed the culture medium from 1-Step medium to G1/G2 medium; the remaining 772 IVF-ET cycles in 661 patients were per- formed using G1/G2 medium. Of these cycles, 168 IVF-ET cycles (156 day 3 IVF-ET cycles +12 day 5 IVF-ET cycles) in 159 patients used cell culture dishes, while 628 IVF-ET cycles (539 day 3 IVF-ET cycles + 89 day 5 IVF-ET cycles) in 514 patients used GPS dishes. In the early stage of the study, we found that using the GPS dish had a beneficial effect on clinical outcomes, and after confirming a significant improvement in pregnancy outcomes in the GPS dish group, we completely changed to GPS dishes. The ultimate goal of our studies is to improve the pregnancy rate in IVF cycles; therefore, we could no longer use the cell culture dishes. This change resulted in the difference of the number of cycles between the cell culture and GPS dish groups.

\section{Ovarian stimulation and oocyte aspiration}

Controlled stimulation for IVF cycles was performed with a mild stimulation protocol using a combination of a gonadotropin-releasing hormone $(\mathrm{GnRH})$ antagonist and gonadotropins. Patients received $150 \mathrm{IU}$ of recombinant follicle-stimulating hormone (Gonal-F; Merck Serono, Darmstadt, Germany) alone as a daily injection from cycle day 3 until the day when human chorionic gonadotropin (hCG) was administered. The GnRH antagonist (Cetrotide, Merck Serono) was initiated on the day when the leading follicle reached a diameter of $14 \mathrm{~mm}$. Ovarian follicular development was monitored by transvaginal ultrasonography. When the leading follicles reached $\geq 18$ $\mathrm{mm}$ in maximum diameter, as detected by sonography, ovulation was induced by injecting $250 \mu \mathrm{g}$ of hCG (Ovidrel, Merck Serono). Oocyte retrieval was performed using 20-gauge ovum aspiration needles (Cook Medical, Bloomington, IN, USA) under standard transvaginal ultrasound guidance 35-36 hours after hCG administration. The luteal phase was supported by progesterone injection or vaginal gel (Crinone, Merck Serono). A serum $\beta$-hCG test was performed about 2 weeks after oocyte retrieval. Clinical pregnancy was confirmed by the visualization of a gestational sac. Ongoing pregnancy was defined as a pregnancy that was maintained for over 20 weeks of gestation.

\section{Embryo culture in vitro}

One-Step (Origio, Malov, Denmark) and G1/G2 media (Vitrolife, Göteborg, Sweden) were employed in IVF-ET cycles. The culture dishes for IVF and embryo culture were prepared and incubated in a humid-type incubator (HERAcell 150i; Thermo Scientific, Waltham, MA, USA) overnight to achieve an optimal pH of 7.2-7.3. Fertilized oocytes were individually cultured in $20-\mu \mathrm{L}$ drops of the culture medium covered in oil for 3-5 days until transfer, in $6.0 \% \mathrm{CO}_{2}, 5 \% \mathrm{O}_{2}$ and $89.0 \% \mathrm{~N}_{2}$, in a dry-type incubator (Miri; ESCO, New Haven, CT, USA). The embryos were cultured in a cell culture dish without outer-well medium (dry culture condition) or in a $\mu$-drop GPS dish with out- 
er-well medium (humid culture condition). In day 5 IVF-ET cycles of the cell culture dish and GPS dish groups, dish change was performed on day 3 by transferring the embryos to a new culture dish prepared on day 2 , to inhibit osmotic changes induced by evaporation and to serve as a new culture medium.

\section{Estimation of osmolality changes in media}

Osmotic changes in micro-drops $(20 \mu \mathrm{L})$ of medium covered in oil and with or without outer-well medium supplementation were compared in the following types of culture dishes: cell culture (Corning Inc., Corning, NY, USA), $\mu$-droplet culture (Vitrolife), microwell culture (DNP, Kashiwa, Japan), and $\mu$-drop GPS (LifeGlobal, Brussels, Belgium) (Figure 1). Ham's F-10 (Gibco, Grand Island, NY, USA) and G1 (Vitrolife) were used to investigate changes in osmolality. The basic osmolality of the media was about 280 and $275 \mathrm{mOsm} / \mathrm{kg}$, respectively. The osmolality of media was estimated with a micro-osmometer (Advanced, Norwood, MA, USA), after 3 days of incubation in the dry-type incubator.

\section{Embryo sequential scoring}

The quality of embryos was daily evaluated and scored according to the developmental stage and speed, as well as the shape of blastomeres and degree of fragmentation (Figure 2). The embryo grades and scores were as follows: grade 1 ( +5 points), no fragmentation

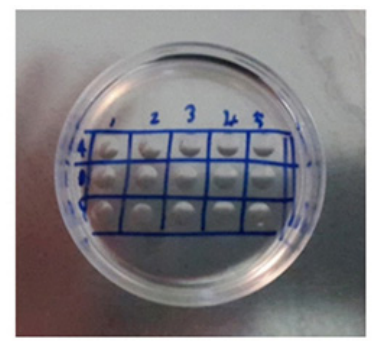

Cell culture (Corning, dry culture)

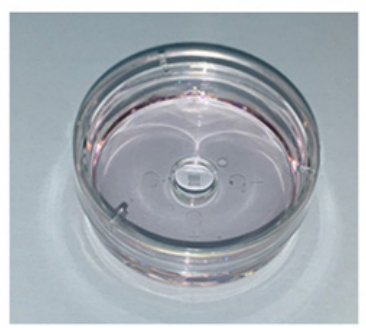

Microwell culture (DNP, humid culture)

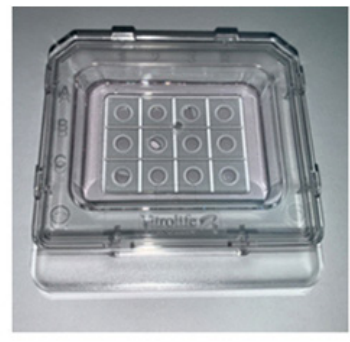

$\mu$-droplet culture (Vitrolife, humid culture)

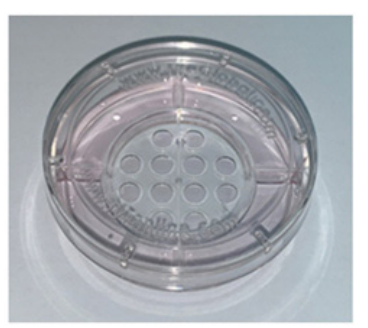

$\mu$-drop GPS

(Life Global, humid culture)
Figure 1. Various culture dishes were used to compare osmotic changes of $\mu$-drop medium covered in oil and with (humid culture) or without outer well medium (dry culture) after 3 days of incubation in a dry-type incubator. with equal-sized blastomeres; grade 2 (+4 points), < 10\% fragmentation with equal-sized blastomeres or no fragmentation with unequal-sized blastomeres; grade 3 (+3 points), 10\% $\leq$ fragmentation $<25 \%$; grade 4 ( +2 points), $25 \% \leq$ fragmentation $<50 \%$; grade 5 (+1 point), $\geq 50 \%$ fragmentation; arrested embryos ( 0 point). When an embryo showed a normal developmental speed and stage, an additional point was given (+1 point), such as two-cell cleavage on day 1 , four-cell stage on day 2 , eight-cell stage on day 3 , or compaction on day 4. It has been reported that fast cleavage speed on day 4 showed an association with high aneuploidy and low blastocyst formation rates. Moreover, in our accumulated data, the pregnancy rate of embryos with a normal cleavage speed was higher than highspeed embryos or low-speed embryos. The quality of blastocysts were evaluated separately in terms of the inner cell mass and trophectoderm; each cell type was classified as grade $A$ to $C$, and points were given according to the grades, as follows; $A$ ( +3 points), $B(+2$ points) and $C$ (+1 point). The embryos with the highest cumulative scores were selected for transfer.

\section{Statistical analysis}

Statistical analysis was performed with SPSS ver. 11.0 (SPSS Inc., Chicago, IL, USA). Means and standard deviations were calculated for all variables. The Student $t$-test was employed to analyze differences in patients' age, endometrial thickness, number of oocytes retrieved, scores of embryos, and number of embryos transferred between the dry and humid culture groups. Differences in clinical outcomes between the two groups were analyzed by the chi-square test, and $p$-values $<0.05$ were considered to indicate statistical significance.

\begin{tabular}{|l|c|c|c|c|c|}
\hline Embryo quality & Day 1 & Day 2 & Day 3 & Day 4 & Day 5 \\
\hline 2-Cell cleavage & +1 & & & & \\
\hline Grade 1 & & +5 & +5 & & \\
\hline Grade 2 & & +4 & +4 & & \\
\hline Grade 3 & & +3 & +3 & & \\
\hline Grade 4 & & +2 & +2 & & \\
\hline Grade 5 & & +1 & +1 & & \\
\hline Arrest & & 0 & 0 & 0 & 0 \\
\hline 4-Cell stage & & +1 & & & \\
\hline 8-Cell stage & & & +1 & & \\
\hline Compaction & & & & +1 & \\
\hline Expanded & & & & & +3 \\
\hline Expanding & & & & & +1 \\
\hline Early & & & & & +3 \\
\hline AA (ICM/trophectoderm) & & & & & +2 \\
\hline AB, BA & & & & & +1 \\
\hline BB & & & & & \\
\hline
\end{tabular}

Figure 2. Embryo sequential scoring for evaluation of embryo quality. ICM, inner cell mass. 


\section{Results}

1. Osmotic changes in media according to the type of culture dish after 3 days of incubation in a dry-type incubator

Osmotic changes in 20- $\mu \mathrm{L}$ droplets of Ham's F-10 (280 mOsm) and G1 (275 mOsm) media according to the various types of culture dishes were compared after 3 days of incubation in the dry-type incubator (Table 1). The osmolality of Ham's F-10 in the cell culture dishes (301.1 mOsm) in the dry-type incubator was higher than that (286.1 $\mathrm{mOsm}$ ) in the humid-type incubator, although the dishes were covered in $6 \mathrm{~mL}$ of oil. Compared to the cell culture dishes with dry culture conditions (without outer-well medium, $301.1 \mathrm{mOsm}$ ), the humid culture conditions (with outer-well medium) using the $\mu$-droplet culture (290.4 mOsm), Microwell culture (285.3 mOsm) and $\mu$-drop GPS (287.2 mOsm) dishes showed lower osmolality. The osmolality of the G1 medium in the cell culture dishes (293.7 mOsm) and $\mu$-drop GPS dishes without outer-well medium (293.6 mOsm) was higher than that in the $\mu$-drop GPS dishes with outer-well medium ( $285.0 \mathrm{mOsm}$ ) after 3 days of incubation in the dry-type incubator. Although there was no difference in the osmotic change between the GPS dish and the other dishes supplemented with outer well medium, we selected the $\mu$-drop GPS dishes to use for human embryo culture because of their suitability for our culture system.

\section{Clinical outcomes of the first (cell culture dish, dry culture)} and second day 3 IVF-ET cycles (GPS dish, humid culture) in the same 12 patients using 1-Step (Origio) medium

As shown in Table 1, supplementation of outer-well medium (humid culture condition) was efficient for maintaining the osmolality of media in the dry-type incubator. To investigate the possible beneficial effects of the humid culture condition, in the same 12 patients, the first IVF-ET cycle was performed using the cell culture dishes and the second IVF-ET cycle was performed using the GPS dishes (Table 2).

There were no significant differences in the characteristics of the

Table 1. Osmotic changes in media according to the type of culture dishes after 3 days of incubation in a dry type incubator

\begin{tabular}{|c|c|c|c|c|c|}
\hline Culture dish & Incubator type & Media (mOsm/kg) & Cover oil (mL) & Outer well media $(\mathrm{mL})$ & Osmolality (mOsm $/ \mathrm{kg})$ \\
\hline Cell culture (Corning) & Humid & Ham's F-10 (280) & 6 & 0 & 286.1 \\
\hline Cell culture (Corning) & Dry & Ham's F-10 (280) & 6 & 0 & 301.1 \\
\hline Microwell culture (DNP) & Dry & Ham's F-10 (280) & 0.03 & 2 & 285.3 \\
\hline $\mu$-drop GPS (LifeGlobal) & Dry & Ham's F-10 (280) & 2 & 4 & 287.2 \\
\hline Cell culture (Corning) & Dry & G1 (275) & 6 & 0 & 293.7 \\
\hline$\mu$-drop GPS (LifeGlobal) & Dry & G1 (275) & 2 & 4 & 285.0 \\
\hline
\end{tabular}

Humid, HERAcell 150i incubator; Dry, ESCO Miri R incubator.

Table 2. Clinical outcomes of the first (cell culture dish, dry culture) and second (GPS dish, humid culture) day 3 IVF-ET cycles in the same 12 patients using 1-step (Origio) medium

\begin{tabular}{|c|c|c|c|c|c|c|c|}
\hline \multirow{2}{*}{ 1-step medium/day 3 ET (dry-type incubator) } & \multicolumn{3}{|c|}{ First IVF cycle (cell culture dish) } & \multicolumn{3}{|c|}{ Second IVF cycle (GPS dish) } & \multirow{2}{*}{$p$-value } \\
\hline & ICSI & cIVF & Total & ICSI & cIVF & Total & \\
\hline No. of IVF cycles & 8 & 4 & 12 & 9 & 3 & 12 & \\
\hline Mean age (yr) & 40.1 & 35 & $38.4 \pm 4.9$ & 40.3 & 33.3 & $38.5 \pm 4.9$ & 0.467 \\
\hline Mean endometrium thickness (mm) & 9.9 & 9.3 & $9.7 \pm 1.7$ & 9.8 & 11.1 & $10.1 \pm 2.1$ & 0.300 \\
\hline Mean no. of oocytes aspirated & 4.5 & 7.7 & $5.5 \pm 3.9$ & 5.4 & 6 & $5.5 \pm 2.6$ & 0.500 \\
\hline 2PN oocytes (\%) & $26 / 36(72.2)$ & $17 / 31(54.8)$ & $43 / 67(71.6)$ & $29 / 49(59.1)$ & $14 / 18(77.7)$ & $43 / 67(74.1)$ & 0.924 \\
\hline Mean no. of embryos transferred & 1.7 & 2.0 & $1.8 \pm 0.3$ & 2.0 & 2.0 & $2.0 \pm 0.0$ & 0.076 \\
\hline Mean score of embryos transferred & 7.3 & 8.3 & $7.6 \pm 2.2$ & 6.4 & 8.0 & $6.8 \pm 2.7$ & 0.211 \\
\hline Clinical pregnancy cycle (\%) & $3(37.5)$ & $1(25.0)$ & $4(30.7)$ & $4(44.4)$ & $2(66.6)$ & $6(50.0)$ & 0.678 \\
\hline Abortion cycle (\%) & $3(37.5)$ & $1(25.0)$ & $4(30.7)$ & 0 & 0 & 0 & 0.100 \\
\hline Ongoing pregnancy (\%) & 0 & 0 & 0 & $4(44.4)$ & $2(66.6)$ & $6(50.0)^{\mathrm{a})}$ & 0.018 \\
\hline
\end{tabular}

Values are presented as mean \pm standard deviation or number (\%).

Dry culture, without outer well medium; Humid culture, with outer well medium; IVF, in vitro fertilization; ET, embryo transfer; ICSI, intracytoplasmic sperm injection; cIVF, conventional IVF; PN, pronuclei.

${ }^{\text {a) }} p<0.05$. 
same 12 patients between the first and second IVF-ET cycles, because the second IVF-ET cycles were performed within 6 months after the completion of the first IVF cycles. Although the rates of clinical pregnancy and abortion (50.0\% and $0 \%$ ) in the second IVF-ET cycles were more favorable than those ( $30.7 \%$ and $30.7 \%$, respectively) in the first IVF-ET cycles, the difference was not statistically significant. However, the ongoing pregnancy rate $(50.0 \%)$ of the second IVF-ET cycles was significantly higher than the rate $(0 \%, p<0.05)$ of the first IVF-ET cycles. When the two IVF cycle groups were subdivided into intracytoplasmic sperm injection (ICSI) and conventional IVF cycle groups, no differences were found in the characteristics of patients and clinical outcomes between the subgroups of the first and second IVF-ET cycle groups.

\section{Clinical outcomes of day 3 IVF-ET cycles in the cell culture} dish (dry culture) and GPS dish (humid culture) groups using G1/G2 medium (Vitrolife)

In the day 3 IVF-ET cycles, there were no significant differences in the mean age ( $36.9 \pm 4.4$ and $37.3 \pm 4.2)$, endometrial thickness (10.6 \pm 2.4 and $10.4 \pm 2.0 \mathrm{~mm})$, score of transferred embryos $(9.1 \pm 2.3$ and $8.9 \pm 2.4)$ and fertilization rate (67.4 and $69.4 \%$ ) between the dry (156 cycles) and humid culture (539 cycles) groups (Table 3). However, the numbers of oocytes retrieved $(8.4 \pm 4.4)$ and transferred embryos $(2.0 \pm 0.4)$ in the dry culture group were significantly higher than those in the humid culture group $(7.3 \pm 4.0$ and $1.9 \pm 0.3$, respectively, $p<0.05)$. Nevertheless, the rates of abortion and ongoing pregnancy in the humid culture group (1.5\% and $49.5 \%)$ were significantly more favorable than those in the dry culture group $(8.3 \%$ and $37.8 \%$, respectively, $p<0.01$ ), although there was no significant difference in the clinical pregnancy rate between the dry (46.2\%) and humid culture (51.0\%) groups. When the two culture groups were subdivided into ICSI and conventional IVF cycle groups, the characteristics of patients and clinical outcomes of the subgroups showed a similar pattern to the total IVF cycles between the two culture groups.

\section{Clinical outcomes of day 5 IVF-ET cycles in the cell culture dish (dry culture) and GPS dish (humid culture) groups using G1/G2 medium}

In the day 5 IVF-ET cycles, there were also no differences in the mean age (36.1 \pm 3.9 and $36.3 \pm 3.4$ years, respectively), endometrial thickness (10.8 \pm 1.7 and $10.6 \pm 2.1 \mathrm{~mm}$, respectively), the number $(1.0 \pm 0.0$ and $1.0 \pm 0.2$, respectively) and score (16.5 \pm 1.8 and $15.3 \pm 3.0$, respectively) of embryos transferred, or the fertilization rate $(77.7 \%$ and $76.2 \%$, respectively) between the dry ( 12 cycles) and humid culture (89 cycles) groups (Table 4). However, the number of oocytes aspirated $(12.3 \pm 4.7)$ in the dry culture group was significantly higher than that in the humid culture group $(9.9 \pm 3.4$, $p<0.05)$. The abortion rate in the humid culture group (2.2\%) was significantly lower than that in the dry culture group $(25.0 \%$, $p<0.01$ ), but no statistically significant difference was observed in the rates of clinical and ongoing pregnancy between the dry $(50 \%$ and $25.0 \%$ ) and humid culture groups (59.5\% and $57.3 \%$, respectively), most likely due to the small number of cycles in the dry culture group. When the day 5 IVF-ET cycles in the two culture groups were also subdivided into ICSI and conventional IVF cycle groups, the characteristics of patients and clinical outcomes of the subgroups showed a similar trend to the total IVF cycles between the two cul-

Table 3. Clinical outcomes of day 3 IVF-ET cycles in cell culture dish (dry culture) and GPS dish (humid culture) groups using G1/G2 medium (Vitrolife)

\begin{tabular}{|c|c|c|c|c|c|c|c|}
\hline \multirow{2}{*}{ G1/G2 media/day 3 ET (dry-type incubator) } & \multicolumn{3}{|c|}{ Cell culture dish (dry culture) } & \multicolumn{3}{|c|}{ GPS dish (humid culture) } & \multirow{2}{*}{$p$-value } \\
\hline & ICSI & cIVF & Total & ICSI & CIVF & Total & \\
\hline No. of IVF cycles & 82 & 74 & 156 & 236 & 303 & 539 & \\
\hline Mean age (yr) & 37.3 & 36.6 & $36.9 \pm 4.4$ & 38.2 & 36.5 & $37.3 \pm 4.2$ & 0.434 \\
\hline Mean endometrium thickness (mm) & 10.6 & 10.6 & $10.6 \pm 2.4$ & 14.2 & 10.5 & $10.4 \pm 2.0$ & 0.354 \\
\hline Mean no. of oocytes aspirated & $7.9^{\mathrm{a})}$ & $8.9^{\mathrm{a})}$ & $8.4 \pm 4.4^{\mathrm{a})}$ & 6.4 & 8.0 & $7.3 \pm 4.0$ & 0.003 \\
\hline 2PN oocytes (\%) & $411(63.1)$ & $471(71.5)$ & $882(67.4)$ & $992(65.7)$ & $1,737(71.7)$ & $2,729(69.4)$ & 0.177 \\
\hline Mean no. of embryos transferred & $1.9^{\mathrm{a})}$ & 2.0 & $2.0 \pm 0.4^{\mathrm{a})}$ & 1.8 & 2.0 & $1.9 \pm 0.3$ & 0.013 \\
\hline Mean score of embryos transferred & 8.8 & 9.4 & $9.1 \pm 2.3$ & 8.4 & 9.2 & $8.9 \pm 2.4$ & 0.273 \\
\hline Clinical pregnancy cycle (\%) & $36(43.9)$ & $36(48.6)$ & $72(46.2)$ & $103(43.6)$ & $172(56.7)$ & $275(51.0)$ & 0.337 \\
\hline Abortion cycle (\%) & $7(8.5)^{a)}$ & $6(8.1)^{a)}$ & $13(8.3)^{\mathrm{a})}$ & $3(1.3)$ & $5(1.7)$ & $8(1.5)$ & $<0.001$ \\
\hline Ongoing pregnancy (\%) & $29(35.3)$ & $30(40.5)$ & $59(37.8)$ & $100(42.3)$ & $167(55.1)^{\mathrm{a})}$ & $267(49.5)^{\mathrm{a})}$ & 0.012 \\
\hline
\end{tabular}

Values are presented as mean \pm standard deviation or number (\%).

IVF, in vitro fertilization; ET, embryo transfer; Dry culture, without outer well medium; Humid culture, with outer well medium; ICSI, intracytoplasmic sperm injection; cIVF, conventional IVF; PN, pronuclei.

${ }^{\text {a) }} p<0.05$. 
ture groups.

\section{Discussion}

When the osmolality of the medium increases above a certain threshold, embryo development is compromised [1,5] and apoptosis is increased [19]. Hyperosmolality ( $>300 \mathrm{mOsm}$ ) was shown to have a detrimental effect on preimplantation mouse embryo development in previous studies [4-6]. However, paradoxically, the osmolality of oviductal fluid is about 340 mOsm [2,20], which is high enough to impair mouse embryo development in vitro. However, unlike in vitro, mouse zygotes have no difficulty developing in vivo, which suggests that an unknown mechanism may exist that helps them overcome the detrimental effect of hyperosmolality in vivo [1].

One possible mechanism is the use of various amino acids as organic osmolytes by embryos. The addition of various organic osmolytes, including taurine [21-24], hypotaurine [25], and glutamine [26], were beneficial for embryo culture. Glycine has also been shown to protect mouse [3] and rabbit embryos [27] against the effects of high $\mathrm{NaCl}$ levels. Indeed, over $70 \%$ of zygotes developed to the blastocyst stage when $1 \mathrm{mM}$ glutamine was present at $310 \mathrm{mOsm}$, but only about $10 \%$ did so in the absence of glutamine [1]. At $310 \mathrm{mOsm}$ of KSOM medium in the absence of glycine, over $80 \%$ of outbred mouse zygotes were arrested at the two-cell stage. However, in the presence of glycine, $60 \%$ of the zygotes developed to the blastocyst stage [12]. Furthermore, more blastocysts formed when bovine zygotes were cultured in a 247 mOsm medium (34.6\%) than in a 286 mOsm medium (17.0\%) in the presence of $1 \mathrm{mM}$ glycine [28]. The beneficial effect of organic osmolytes can be explained by previous findings that amino acids, including osmolytes, are present in oviductal and uterine fluid [29-31] and carry out various physiological functions in the preimplantation embryo, including ATP production [32], ammonium detoxification [33,34] and maintaining the redox balance [35].

Baltz and Tartia [36] suggested a possible answer for the question "Why did lower osmolarity support embryo development in culture?" They proposed that the osmolality in the in vivo environment of early preimplantation embryos may be lower than in blood plasma (280 mOsm). This possibility conflicts with previous reports that the osmolality of oviductal fluid is about 340 mOsm [2,20]. Although the oviduct is the major in vivo environment for early-stage embryos, the follicle can be seen as the birthplace of the oocyte and embryo. In mice, a large amount of follicular fluid moves into the ampulla of the oviduct with all ovulated oocytes via the ovarian bursa. In human, immediately after ovulation, a small amount of follicular fluid flows weakly from the ovarian wall to the ampulla of the oviduct to help the sliding movement of the oocyte. This means that follicular fluid may play an important role in determining the oviductal environment for early-stage embryos. In our preliminary test (data not published), we compared the osmolality of human follicular fluid and blood serum. Interestingly, the osmolality of follicular fluid (275.3 mOsm) was lower than that of serum ( $282.0 \mathrm{mOsm})$. There are still no reports about the osmolality of human oviductal fluid, so further research on the osmolality of the in vivo environment of human preimplantation embryos is required. Nevertheless, interestingly, current commercial culture media already have lower osmolality (260-270 mOsm) than the older generation of media (280-295 mOsm) [36].

In the present study, after 3 days of incubation, even under an oil

Table 4. Clinical outcomes of day 5 IVF-ET cycles in cell culture dish (dry culture) and GPS dish (humid culture) groups using G1/G2 medium (Vitrolife)

\begin{tabular}{|c|c|c|c|c|c|c|c|}
\hline \multirow{2}{*}{ G1/G2 media/day 5 ET (dry-type incubator) } & \multicolumn{3}{|c|}{ Cell culture dish (dry culture) } & \multicolumn{3}{|c|}{ GPS dish (humid culture) } & \multirow{2}{*}{$p$-value } \\
\hline & ICSI & cIVF & Total & ICSI & cIVF & Total & \\
\hline No. of IVF cycles & 5 & 7 & 12 & 28 & 61 & 89 & \\
\hline Mean age (yr) & 36.6 & 35.7 & $36.1 \pm 3.9$ & 36.8 & 36 & $36.3 \pm 3.4$ & 0.846 \\
\hline Mean endometrium thickness (mm) & 9.84 & 11.5 & $10.8 \pm 1.7$ & 10.8 & 10.5 & $10.6 \pm 2.1$ & 0.701 \\
\hline Mean no. of oocytes aspirated & $12.8^{\mathrm{a})}$ & 12 & $12.3 \pm 4.7$ & 9 & 10.2 & $9.9 \pm 3.4$ & 0.026 \\
\hline 2PN oocyte (\%) & $51(79.6)$ & $64(76.1)$ & $115(77.7)$ & $190(75.3)$ & $480(76.5)$ & $670(76.2)$ & 0.773 \\
\hline Mean no. of embryos transferred & 1.0 & 1.0 & $1.0 \pm 0.0$ & 1.0 & 1.0 & $1.0 \pm 0.2$ & 0.459 \\
\hline Mean score of embryos transferred & 16.4 & 16.6 & $16.5 \pm 1.8$ & 14.5 & 15.6 & $15.3 \pm 3.0$ & 0.168 \\
\hline Clinical pregnancy cycle (\%) & $3(60.0)$ & $3(42.8)$ & $6(50.0)$ & $14(50.0)$ & $39(63.9)$ & $53(59.5)$ & 0.750 \\
\hline Abortion cycle (\%) & $1(20.0)$ & $2(28.5)$ & $3(25.0)^{a)}$ & 0 & $2(3.3)$ & $2(2.2)$ & 0.006 \\
\hline Ongoing pregnancy (\%) & $2(40.0)$ & $1(14.2)$ & $3(25.0)$ & $14(50.0)$ & $37(60.6)$ & $51(57.3)$ & 0.072 \\
\hline
\end{tabular}

Values are presented as mean \pm standard deviation or number (\%).

IVF, in vitro fertilization; ET, embryo transfer; Dry culture, without outer well medium; Humid culture, with outer well medium; ICSI, intracytoplasmic sperm injection; CIVF, conventional IVF; PN, pronuclei. a) $p<0.05$. 
layer, the osmolality of media in the dry culture condition (301.1 mOsm) was higher than that of media in the humid culture condition (285.3-290.4 mOsm) in the dry-type incubator. Gasperin et al. [37] also reported that in the absence of an oil layer, the addition of water in the four-well dish central hole reduced the osmolality of medium (294 mOsm) after 24 hours of incubation compared to the osmolality of control dishes without water ( $305 \mathrm{mOsm}$ ) in a humid-type incubator. These results indicate that osmolality can increase in both dry- and humid-type incubators. Therefore, both an oil layer and extra medium or water supplementation are essential for maintaining the osmolality of the medium by inhibiting evaporation in in vitro culture conditions.

In the present study, in day $5 \mathrm{ET}$ cycles, the rates of clinical and ongoing pregnancy in the humid culture group were higher than the rates in the dry culture group. Similarly, no meaningful difference was found in the clinical and ongoing pregnancy rates of the humid culture groups in day $5 \mathrm{ET}$ cycles ( $57 \%$ and $52 \%$ ) between the previous [18] and the present study (59.5\% and 57.3\%), even though there was a large difference in the mean age of female patients between the two studies (28 vs. 36 years, respectively). The first possible reason is medium renewal. We performed medium renewal by exchanging G1 to G2 medium on day 3, but Fawzy et al. [18] did not perform medium renewal for 5 days. Culture for 5 days without medium renewal could degrade the organic osmolytes, and this degradation may augment the negative influence of this osmolality shift on embryo development. In addition, medium renewal may not only reduce the accumulation of metabolites such as ammonium, but could also supply fresh nutrients for embryo development. The second possible reason relates to the humid culture condition. In the present study, for the preparation of the humid culture condition, we added $6 \mathrm{~mL}$ of medium into the outer well of GPS culture dishes (LifeGlobal), while Fawzy et al. [18] used petri dishes containing 10 $\mathrm{mL}$ of water. In the preliminary experiment, we also used petri dishes containing water, but this was not efficient to inhibit the increase of osmolality in the medium compared to direct medium supplementation in the outer well of the culture dish.

Fawzy et al. [18] reported that the blastulation rate of human embryos was significantly higher in the humid culture group (73\%) than in the dry culture group $(51 \%, p<0.05)$. Gasperin et al. [37] reported a similar trend, according to which the blastocyst rate of bovine embryos was higher in the humid culture group $(29.7 \%, p<0.05)$ than in the dry culture group (16.2\%). Unlike the above results, in the present study, there was no significant difference in the blastulation rate between the dry (56.5\%) and humid culture (55.5\%) groups. Of particular note, the blastulation rate of the humid culture group (55.5\%) was markedly lower than the rate (73\%) reported in the other study [18]. This difference may have resulted from the large gap in the mean age of female patients between the other study (28 years) and the present study (36 years). The age of female patients is widely considered as a critical factor for determining the quality of oocytes, the grade of embryos, and the success of pregnancy in IVF-ET cycles. Therefore, the relatively low blastulation rate in the humid culture group in the present study may be explained by the relatively old age of patients compared to the previous study [18].

There was no significant difference in the development of early-stage mouse embryos cultured between the high-osmolality (310-330 mOsm) and low-osmolality (270-290 mOsm) media. However, in the development of late-stage embryos, the embryos cultured in the low-osmolality medium showed a significantly higher blastocyst formation rate than those cultured in the high-osmolality medium [6]. A similar result was observed in porcine embryos cultured in vitro [8]. These results suggest that late-stage embryos are more susceptible to osmotic changes than early-stage embryos. In the present study, a similar pattern in response to osmotic changes in human embryos was observed, although we did not observe a significant difference in the blastocyst formation rate between the high-osmolality ( $56.5 \%$, dry culture) and low-osmolality $(55.5 \%$, humid culture) culture groups. However, in day 3 ET cycles, involving relatively early-stage embryos compared to day $5 \mathrm{ET}$ cycles, the transferred embryos showed a small difference in pregnancy outcomes between the high-and low-osmolality culture groups. The differences in the rates of pregnancy, abortion, and ongoing pregnancy were $4.8 \%, 6.8 \%$, and $11.7 \%$, respectively. In contrast, in day $5 \mathrm{ET}$ cycles, the differences in these rates in the late-stage embryos were $9.5 \%, 22.8 \%$, and $32.3 \%$, respectively. This result is consistent with the previous finding that late-stage embryos are more susceptible to osmotic changes $[6,8]$.

When a cell shrinks in hyperosmotic conditions, inorganic ions $\left(\mathrm{Na}^{+}, \mathrm{K}^{+}\right)$are accumulated via osmotically regulated ion transporters [38]. However, in this case, cells accumulate organic osmolytes intracellularly to replace a portion of the inorganic ions [39-41]. High ionic strength disrupts cell functions, while in contrast, a high concentration of organic osmolytes is not toxic [39,42]. The beneficial action of organic osmolytes was proven by a report that in a hyperosmotic medium (310 mOsm), the blastocyst formation rate of mouse embryos cultured in the presence of $1 \mathrm{mM}$ glycine was significantly more favorable than that of embryos cultured in the absence of glycine [12].

We used G1/G2 medium (Vitrolife), which contains 135-145 $\mu \mathrm{M}$ glycine [43], a concentration that is much lower than the effective concentration of $1 \mathrm{mM}$ in previous studies [12,28]. In the present study, the osmolality of G1 medium was $275 \mathrm{mOsm}$, which increased to 293.7 and $285.0 \mathrm{mOsm}$ in the dry and humid culture conditions, respectively, after 3 days of incubation. The better clinical outcomes 
in the humid culture condition than the dry culture condition might have resulted from the low osmolality of the medium in the humid culture condition. Due to the maintenance of low osmolality in the culture medium, glycine probably did not have the chance to act as an organic osmolyte. Instead, the higher osmotic change in the dry culture condition might have increased the concentration of intracelIular ionic osmolytes, which are detrimental for embryo development. Moreover, a portion of the ionic osmolytes could not be replaced completely with organic osmolytes because of the low concentration of glycine [43].

In conclusion, the dry culture condition showed a higher osmotic change in the medium than the humid culture condition, even though the media were covered in oil. Hyperosmotic changes showed a detrimental effect on clinical outcomes in human IVF-ET cycles. This hyperosmotic stress could be alleviated by supplementation with outer-well medium to maintain the optimal osmolality of medium, which resulted in the improvement of clinical outcomes in IVF-ET cycles. We are preparing a follow-up study to investigate relationships between the osmolality of culture media and specific patterns of gene expression in porcine embryos.

\section{Conflict of interest}

No potential conflict of interest relevant to this article was reported.

\section{ORCID}

Hee-Jun Chi
Jun-Sang Park
Chang-Seok Yoo
Su-Jin Kwak
Ho-Jeong Son
Seok-Gi Kim
Chae-Hee Sim
Kyeong-Ho Lee
Deog-Bon Koo

https://orcid.org/0000-0001-8688-5820 https://orcid.org/0000-0003-3033-2789 https://orcid.org/0000-0002-8477-3435 https://orcid.org/0000-0002-7538-6919 https://orcid.org/0000-0003-4763-496X https://orcid.org/0000-0002-5893-5920 https://orcid.org/0000-0003-2174-9179 https://orcid.org/0000-0002-4231-3780 https://orcid.org/0000-0001-7825-9598

\section{Author contributions}

Conceptualization: HJC. Data curation: HJC, CSY, SJK, HJS, SGK. Formal analysis: HJC, JSP. Funding acquisition: CHS, KHL. Methodology: HJC. Project administration: HJC. Visualization: HJC. Writing-original draft: HJC. Writing-review \& editing: HJC, DBK

\section{References}

1. Dawson KM, Baltz JM. Organic osmolytes and embryos: substrates of the Gly and beta transport systems protect mouse zygotes against the effects of raised osmolarity. Biol Reprod 1997; 56:1550-8.

2. Van Winkle L, Haghighat N, Campione AL. Glycine protects preimplantation mouse conceptuses from a detrimental effect on development of the inorganic ions in oviductal fluid. J Exp Zool 1990;253:215-9.

3. Biggers JD, Lawitts JA, Lechene CP. The protective action of betaine on the deleterious effects of $\mathrm{NaCl}$ on preimplantation mouse embryos in vitro. Mol Reprod Dev 1993;34:380-90.

4. Hay-Schmidt A. The influence of osmolality on mouse two-cell development. J Assist Reprod Genet 1993;10:95-8.

5. Richards T, Wang F, Liu L, Baltz JM. Rescue of postcompaction-stage mouse embryo development from hypertonicity by amino acid transporter substrates that may function as organic osmolytes. Biol Reprod 2010;82:769-77.

6. Swain JE, Cabrera L, Xu X, Smith GD. Microdrop preparation factors influence culture-media osmolality, which can impair mouse embryo preimplantation development. Reprod Biomed Online 2012;24:142-7.

7. Miyoshi K, Funahashi H, Okuda K, Niwa K. Development of rat one-cell embryos in a chemically defined medium: effects of glucose, phosphate and osmolarity. J Reprod Fertil 1994;100:21-6.

8. Beckmann LS, Day BN. Effects of media $\mathrm{NaCl}$ concentration and osmolarity on the culture of early-stage porcine embryos and the viability of embryos cultured in a selected superior medium. Theriogenology 1993;39:611-22.

9. Nguyen VT, Kure-bayashi S, Harayama H, Nagai T, Miyake M. Stage-specific effects of the osmolarity of a culture medium on the development of parthenogenetic diploids in the pig. Theriogenology 2003;59:719-34.

10. Liu Z, Foote RH. Sodium chloride, osmolyte, and osmolarity effects on blastocyst formation in bovine embryos produced by in vitro fertilization (IVF) and cultured in simple serum-free media. J Assist Reprod Genet 1996;13:562-8.

11. Herrick JR, Bond JB, Magarey GM, Bateman HL, Krisher RL, Dunford SA, et al. Toward a feline-optimized culture medium: impact of ions, carbohydrates, essential amino acids, vitamins, and serum on development and metabolism of in vitro fertilization-derived feline embryos relative to embryos grown in vivo. Biol Reprod 2007;76:858-70.

12. Hadi T, Hammer MA, Algire C, Richards T, Baltz JM. Similar effects of osmolarity, glucose, and phosphate on cleavage past the 2-cell stage in mouse embryos from outbred and F1 hybrid females. 
Biol Reprod 2005;72:179-87.

13. Brocker C, Thompson DC, Vasiliou V. The role of hyperosmotic stress in inflammation and disease. Biomol Concepts 2012;3:34564.

14. Burg MB, Ferraris JD, Dmitrieva NI. Cellular response to hyperosmotic stresses. Physiol Rev 2007;87:1441-74.

15. Reinehr R, Haussinger D. Hyperosmotic activation of the CD95 death receptor system. Acta Physiol (Oxf) 2006;187:199-203.

16. Swain JE. Decisions for the IVF laboratory: comparative analysis of embryo culture incubators. Reprod Biomed Online 2014;28:53547.

17. Geraghty RJ, Capes-Davis A, Davis JM, Downward J, Freshney RI, Knezevic I, et al. Guidelines for the use of cell lines in biomedical research. Br J Cancer 2014;111:1021-46.

18. Fawzy M, AbdelRahman MY, Zidan MH, Abdel Hafez FF, Abdelghafar $\mathrm{H}$, Al-Inany $\mathrm{H}$, et al. Humid versus dry incubator: a prospective, randomized, controlled trial. Fertil Steril 2017;108:277-83.

19. Xie Y, Zhong W, Wang Y, Trostinskaia A, Wang F, Puscheck EE, et al. Using hyperosmolar stress to measure biologic and stress-activated protein kinase responses in preimplantation embryos. Mol Hum Reprod 2007;13:473-81.

20. Borland RM, Hazra S, Biggers JD, Lechene CP. The elemental composition of the environments of the gametes and preimplantation embryo during the initiation of pregnancy. Biol Reprod 1977; 16:147-57.

21. Dumoulin JC, Evers JL, Bras M, Pieters MH, Geraedts JP. Positive effect of taurine on preimplantation development of mouse embryos in vitro. J Reprod Fertil 1992;94:373-80.

22. Spindle A. Beneficial effects of taurine on mouse zygotes developing in protein-free culture medium. Theriogenology 1995;44:761-72.

23. McKiernan SH, Clayton MK, Bavister BD. Analysis of stimulatory and inhibitory amino acids for development of hamster one-cell embryos in vitro. Mol Reprod Dev 1995;42:188-99.

24. Liu Z, Foote RH, Yang X. Development of early bovine embryos in co-culture with KSOM and taurine, superoxide dismutase or insulin. Theriogenology 1995;44:741-50.

25. Barnett DK, Bavister BD. Hypotaurine requirement for in vitro development of golden hamster one-cell embryos into morulae and blastocysts, and production of term offspring from in vitro-fertilized ova. Biol Reprod 1992;47:297-304.

26. Lawitts JA, Biggers JD. Culture of preimplantation embryos. In: Wassarman PM, DePamphilis ML. editors. Guide to techniques in mouse development. San Diego: Academic Press; 1993. p. 153-64.

27. Li J, Foote RH. Effect of inositol and glycine with increasing sodium chloride and constant osmolality on development of rabbit embryos. J Assist Reprod Genet 1995;12:141-6.

28. Herrick JR, Lyons SM, Greene AF, Broeckling CD, Schoolcraft WB,
Krisher RL. Direct and osmolarity-dependent effects of glycine on preimplantation bovine embryos. PLoS One 2016;11:e0159581.

29. Harris SE, Gopichandran N, Picton HM, Leese HJ, Orsi NM. Nutrient concentrations in murine follicular fluid and the female reproductive tract. Theriogenology 2005;64:992-1006.

30. Hugentobler SA, Diskin MG, Leese HJ, Humpherson PG, Watson T, Sreenan JM, et al. Amino acids in oviduct and uterine fluid and blood plasma during the estrous cycle in the bovine. Mol Reprod Dev 2007;74:445-54.

31. Li R, Whitworth K, Lai L, Wax D, Spate L, Murphy CN, et al. Concentration and composition of free amino acids and osmolalities of porcine oviductal and uterine fluid and their effects on development of porcine IVF embryos. Mol Reprod Dev 2007;74:1228-35.

32. Liu LN, Fu TT, Xu XF, Fu C, Fang MJ, Liu Y, et al. Tracing the nitrogen metabolites of glycine using (15)N-glycine and mass spectrometry. Rapid Commun Mass Spectrom 2015;29:645-53.

33. Orsi NM, Leese HJ. Ammonium exposure and pyruvate affect the amino acid metabolism of bovine blastocysts in vitro. Reproduction 2004;127:131-40.

34. Wale PL, Gardner DK. Oxygen affects the ability of mouse blastocysts to regulate ammonium. Biol Reprod 2013;89:75.

35. Lane M, Gardner DK. Mitochondrial malate-aspartate shuttle regulates mouse embryo nutrient consumption. J Biol Chem 2005; 280:18361-7.

36. Baltz JM, Tartia AP. Cell volume regulation in oocytes and early embryos: connecting physiology to successful culture media. Hum Reprod Update 2010;16:166-76.

37. Gasperin BG, Barreta MH, Santos JT, Ferreira R, Neves JP, Oliveira JF, et al. Oil-free culture system for in vitro bovine embryo production. Ital J Anim Sci 2010;9:e32.

38. Hoffmann EK, Lambert IH, Pedersen SF. Physiology of cell volume regulation in vertebrates. Physiol Rev 2009;89:193-277.

39. Yancey PH, Clark ME, Hand SC, Bowlus RD, Somero GN. Living with water stress: evolution of osmolyte systems. Science 1982;217: 1214-22.

40. Lang F, Busch GL, Volkl H. The diversity of volume regulatory mechanisms. Cell Physiol Biochem 1998;8:1-45.

41. Wehner F, Olsen H, Tinel H, Kinne-Saffran E, Kinne RK. Cell volume regulation: osmolytes, osmolyte transport, and signal transduction. Rev Physiol Biochem Pharmacol 2003;148:1-80.

42. Yancey PH. Cell volume regulation: osmolytes, osmolyte transport, and signal transduction. In: Strange K. editor. Cellular and molecular physiology of cell volume regulation. Boca Raton: CRC Press; 1994. p. 81-109.

43. Morbeck DE, Krisher RL, Herrick JR, Baumann NA, Matern D, Moyer T. Composition of commercial media used for human embryo culture. Fertil Steril 2014;102:759-66. 